Revue internationale P.M.E.

Économie et gestion de la petite et moyenne entreprise

\title{
La taille organisationnelle et la satisfaction au travail dans les organisations publiques québécoises
}

\section{Jean-Claude Bernatchez}

Volume 5, numéro 2, 1992

URI : https://id.erudit.org/iderudit/1008138ar

DOI : https://doi.org/10.7202/1008138ar

Aller au sommaire du numéro

Éditeur(s)

Presses de l’Université du Québec

ISSN

0776-5436 (imprimé)

1918-9699 (numérique)

Découvrir la revue

Citer cet article

Bernatchez, J.-C. (1992). La taille organisationnelle et la satisfaction au travail dans les organisations publiques québécoises. Revue internationale P.M.E., 5(2), 41-56. https://doi.org/10.7202/1008138ar

\section{Résumé de l'article}

L'objectif de cette recherche est de saisir j'impact de la taille organisationnelle sur la satisfaction au travail des cadres du secteur public de la santé. Au Québec, les établissements publics possèdent leur conseil d'administration propre dont les pouvoirs sont précisés par une loi habilitante.

Une enquête pan québécoise fut conduite en 1991 auprès d'un échantillon de 691 personnes représentant une population de 10000 cadres.

Dans le réseau public, un dirigeant de PME (500 employés et moins) par rapport à son collègue de la grande entreprise (501 et plus) parvient mieux à inspirer ses subalternes (vision), à faire accepter ses décisions (éthique) et à faire en sorte que ses collaborateurs relèvent les défis de leur fonction (perfectionnement) dans un climat de partage et d'appui (support Interne). Par rapport aux gestionnaires des organisations à taille élevée, les cadres des PME sont plus confiants face à leur avenir professionnel (carrière) et considèrent leur emploi à la fois plus stimulant et exigeant.

Nonobstant la présence d'un régime sophistiqué et étatisé de conditions de travail, la spécificité de la PME publique est réelle au plan de la satisfaction au travail des cadres. 


\title{
La taille organisationnelle et la satisfaction au travail dans les organisations publiques québécoises
}

\author{
Jean-Claude BERNATCHEZ* \\ Université du Québec à Trois-Rivières
}

\begin{abstract}
RÉSUMÉ
L'objectif de cette recherche est de saisir l'impact de la taille organisationnelle sur la satisfaction au travail des cadres du secteur public de la santé. Au Québec, les établissements publics possèdent leur conseil d'administration propre dont les pouvoirs sont précisés par une loi habilitante.
\end{abstract}

Une enquête pan québécoise fut conduite en 1991 auprès d'un échantillon de 691 personnes représentant une population de 10000 cadres.

Dans le réseau public, un dirigeant de PME (500 employés et moins) par rapport à son collègue de la grande entreprise (501 et plus) parvient mieux à inspirer ses subalternes (vision), à faire accepter ses décisions (éthique) et à faire en sorte que ses collaborateurs relèvent les défis de leur fonction (perfectionnement) dans un climat de partage et d'appui (support interne). Par rapport aux gestionnaires des organisations à taille élevée, les cadres des PME sont plus confiants face à leur avenir professionnel (carrière) et considèrent leur emploi à la fois plus stimulant et exigeant.

Nonobstant la présence d'un régime sophistiqué et étatisé de conditions de travail, la spécificité de la PME publique est réelle au plan de la satisfaction au travail des cadres.

* Jean-Claude Bernatchez est professeur au Département d'administration et d'économique de l'Université du Québec à Trois-Rivières. Membre associé du Groupe de recherche en économie et gestion des PME (GREPME), il enseigne les relations du travail. Ses intérêts de recherche concernent principalement le secteur public. L'auteur détient un $\mathrm{Ph}$. D. en relations industrielles. Adresse : GREPME, Université du Québec à Trois-Rivières, C. P. 500, Trois-Rivières, Québec, G9A 5H7. 


\begin{abstract}
The scope of this research is to evaluate the impact of organizational size on work satisfaction among public health managers in Quebec. In fact, each public enterprise is directed by a board which has its own prerogative stemming from a law.

The survey has been conducted in 1991 using a sample of 691 persons. The population concerned is equivalent to 10000 citizens.

In public sector, small and medium business managers (500 employees or less) if compared to their collegues from the large organization (501 or more) are more encline to propose a better future to their subordinates (vision), to make their decisions accepted by employe日s (ethic), to implement training activities based on job realities and to perceive the work site supportive. Finally, managers of small and medium firms are more confident regarding their professional career and se日 their work as satisfying but demanding.

Notwithstanding the work conditions regime elaborated at a national level, the specificity of organizational size is observed and it generates effects on work satisfaction.
\end{abstract}

\title{
RESUMEN
}

El objetivo de esta investigación consiste en comprender el impacto que genera el tamaño de la organización de la empresa en la satisfacción del trabajo de los ejecutivos del sector público de la saluz. En Quebec los establecimientos públicos poseen un consejo de administración propio, cuyos poderes son precisados por una ley de habilitación.

Según la encuesta que hemos realizado en Quebec en 1991 sobre una muestra de 691 personas representado una población de 10000 ejecutivos.

En el sector público, un dirigente de PyME (500 empleados y menos) en relación a su colega de la gran empresa (501 empleados y mas) consigue inspirar mejor a sus subalternos, hacer respectar sus decisiones y lograr que sus colaboradores respondan mejor al reto de superarse en su función, dentro de un clima de participación y apoyo. Además, en relación a los gestores de las grandes firmas, los dirigentes de las PyMES confian más en su futuro profesional (de su carrera) y a la vez consideran su empleo más estimulante.

En consecuencia, la presencia de un régimen sofisticado y burocratizado en las propias condiciones de trabajo da lugar a que la peculiaridad de la PyME pública sea una realidad desde el punto de vista de la satisfacción del trabajo de los ejecutivos. 


\section{Introduction}

La dimension des organisations fait l'objet d'une préoccupation soutenue en sciences administratives. Toutefois, en gestion des ressources humaines et des relations du travail, cette préoccupation est plutôt émergente.

Au Québec, les tailles des établissements du secteur de la santé et des services sociaux sont variées. Telle que démontrée dans cette étude, la dichotomie entre les petites ou moyennes et les grandes entreprises permet de saisir des distinctions intergroupes significatives.

L'objectif de cette recherche est d'observer l'effet de la taille organisationnelle sur la satisfaction au travail des cadres des organisations publiques de la santé et des services sociaux au Québec. Ce secteur compte près d'un millier d'établissements distincts ayant leur conseil d'administration propre, lequel reçoit une délégation de pouvoirs qu'il exerce à l'intérieur des stipulations d'une loi habilitante. Ce réseau d'établissements possède un régime national de normes. Par ailleurs, chaque organisation est libre d'élaborer ses propres règles notamment en matière d'embauche, de perfectionnement et de gestion de carrières.

La satisfaction au travail représente un élément central du succès des organisations sous étude et la main-d'œuvre constitue une large part de leur budget de fonctionnement. De plus, le réseau québécois des affaires sociales est représentée par des établissements dont la dimension fluctue grandement selon les besoins des clientèles. Il est donc intéressant d'étudier cette problématique liée au moral des ressources humaines sous l'angle de la taille organisationnelle. De manière générale, les études sur la satisfaction au travail tiennent peu compte de la dimension des organisations. Cet article vise donc à apporter une contribution à cet égard.

\section{Recension des écrits}

Si la recherche empirique quant à l'effet de la taille sur la satisfaction au travail des cadres en contexte de PME publiques est à l'état embryonnaire, la documentation traitant superficiellement ou en profondeur de la gestion des ressources humaines en PME est plus abondante (MacFarlane, 1977 ; Roxe, 1979 ; Hailes et Hubbard, 1983 ; Arthur, 1987).

Joyce et al. (1990) observent que la préoccupation des gestionnaires de PME à l'égard des ressources humaines est très présente. Ces auteurs notent que parmi les facteurs de succès organisationnels, les dirigeants de PME classent les 
attitudes des employés au deuxième rang immédiatement après la mise en marché. Le moral et la motivation seraient donc une priorité dans les établissements de taille réduite (Benoit-Rousseau, 1990). Ainsi, la gestion des employés se réaliserait sous l'égide de la flexibilité et de la prise de décisions rapides (Buskirk et Vaughn, 1976). Généralement, il est constaté que les difficultés liées aux problèmes du travail, et par conséquent l'insatisfaction, augmentent avec la taille de l'organisation (Mahe de Boislandelle, 1988).

La satisfaction au travail est la résultante de l'adéquation entre les attentes d'un individu à l'égard de son milieu de travail et ce qu'il perçoit réellement en retirer (Le Louarn, 1982). Ce concept avait été antérieurement étudié par Larouche et Delorme (1972) spécialement par la validation d'une échelle de facteurs de satisfaction. Locke (1976) observe que les perceptions des travailleurs varient selon la dimension du travail considérée par ces derniers. Par exemple, un individu peut être satisfait de son horaire et insatisfait de ses choix de carrière. Il s'agit d'une problématique multidimensionnelle. Plusieurs facteurs expliquent la satisfaction au travail comme le perfectionnement personnel, l'autonomie ou les conditions de travail.

Les principales variables identifiables dans la littérature peuvent être regroupées en fonction d'un certain nombre de composantes. La supervision invite à considérer la vision et l'éthique administratives ainsi que le support interne. Les chercheurs tiennent également compte du perfectionnement et des conditions de travail.

Le concept de vision appliqué aux organisations publiques se ramène principalement à l'intrapreneuriat. Pour un individu, cela consiste à développer et appliquer des perceptions qui contribuent au dynamisme d'un système déjà en place. Le développement par l'employeur d'une vision de l'avenir est donc un moyen privilégié pour fournir à ses cadres un guide pour la réflexion et la pratique (Filion, 1990). Toutefois, le risque personnel inhérent à la mise en vigueur d'un comportement intrapreneurial est potentiellement plus élevé dans une organisation de grande taille que dans une autre de taille réduite (Sinetar, 1985). Dans tous les cas, le fait pour un gestionnaire de proposer une vision stimulante du futur représente un élément essentiel de motivation ou de satisfaction (Ket de Vries, 1989).

Ram (1990) rappelle que les organisations de taille réduite sont fréquemment victimes des stéréotypes invoqués à leur égard : elles démontreraient une direction autoritaire, des méthodes de travail inconsidérantes et une discipline injustifiée. Mais, contrairement à l'opinion répandue, il croit qu'à l'intérieur de la PME, la collaboration négociée est souvent au centre de la relation de travail. Implicitement, l'auteur avance que c'est dans un tel contexte que les décisions 
de la direction seront acceptées par ceux qu'elles visent et qu'elles seront en conséquence perçues comme éthiques.

De manière générale, les activités de formation dans les entreprises revêtent un caractère plutôt improvisé et peu planifié (Thompson, 1989). Les petites ou moyennes entreprises n'échapperaient pas à cette règle ; mais les activités formatives qu'elles privilégient seraient rapprochées des opérations courantes (Benoit-Rousseau, 1990). Ainsi, dans les organisations de taille réduite, le perfectionnement des ressources humaines répond à des besoins plutôt ponctuels, mais revêt tout de même une importance stratégique (Blumenthal, 1985). La formation serait perçue par les dirigeants de PME d'une part comme une façon d'augmenter les compétences techniques de l'organisation et, d'autre part, comme un moyen pour maintenir la satisfaction au travail (Hambrick et Crozier, 1984).

Une PME possède un régime de conditions de travail peu formalisé, mais il tend vers une plus grande sophistication avec l'accroissement de la taille de l'entreprise (Arthur, 1987). Les normes internes sont peu sophistiquées (Cohn et Lindberg, 1984) et l'aménagement du temps de travail impose aux ressources humaines une plus grande flexibilité que dans une grande entreprise. Selon Mahe de Boislandelle (1988), la condition de travail du gestionnaire de PME l'oblige systématiquement à appliquer lui-même ce qu'il a conçu :

Si dans une grande entreprise, on peut différencier clairement les rôles entre dirigeant et personnel, il n'en est pas de même dans les PME où le dirigeant est à la fois sujet et objet de la décision. (Mahé de Boislandelle, 1988, p. 10).

La PME utilise donc ses ressources humaines d'une manière différente de la grande entreprise. Par rapport à cette dernière, on y retrouverait peu d'activités stériles ou de pertes de temps, un soutien technique plus externalisé, des niveaux hiérarchiques limités, ainsi qu'une flexibilité supérieure dans la production et des contacts suivis avec la clientèle (Baumback et Lawyer, 1979). Dans un tel contexte, l'employé démontrerait une meilleure compréhension du résultat de son travail sur le succès de l'entreprise (Rimler et Humphreys, 1980).

\section{Méthodologie}

L'objet de l'enquête consistait à relever et analyser les écarts perceptuels observables entre un groupe de cadres œuvrant dans des organisations de taille réduite (PME) par rapport à d'autres œuvrant dans des organisations de grande taille. 
Le répondant fut invité à formuler son opinion sur une échelle de type Likert présentée ainsi :

Exprimez votre degré de satisfaction ou d'insatisfaction (selon le cas) en regard des énoncés suivants :

$$
\begin{aligned}
& 5 \text { = Satisfait(e) } \\
& 4=\text { Plutôt satisfait(e) } \\
& 3 \text { = Autant satisfait(e) qu' 'insatisfait(e) } \\
& 2=\text { Plutôt insatisfait(e) } \\
& 1=\text { Insatisfait(e) }
\end{aligned}
$$

Mon horaire de travail, etc.

La population totale est constituée d'environ 10000 cadres intermédiaires répartis dans l'ensemble des organisations de santé du Québec. Ces dernières sont réparties principalement dans trois catégories d'établissements : les centres hospitaliers, dont la taille est plutôt élevée, et les centres d'accueil ou les centres locaux de santé communautaire, dont la taille est réduite. Des 1600 questionnaires acheminés aux répondants, 693 furent complétés et retournés. De ce nombre, 691 ont été utilisés, ce qui représente un taux de réponse de $43,1 \%$; l'enquête fut réalisée en février 1991.

Le questionnaire comportait une série de questions concernant les perceptions des cadres à l'égard de leur vécu au travail, notamment leur intégration aux valeurs de leur milieu, l'éthique administrative, le perfectionnement, le support interne, leur cheminement de carrière et leurs conditions générales de travail. La formulation des questions fut réalisée à la suite d'une recension d'écrits. Elles furent précisées à l'aide de deux prétests.

La cohérence interne du questionnaire fut vérifiée à l'aide du coefficient Alpha (Novick, 1967). Les corrélations entre les variables soumises à l'analyse s'avérèrent positives et les valeurs Alpha de Cronback se situaient à 0,9704 (échantillon taille large) et 0,9710 (échantillon taille réduite).

La validité du questionnaire fut vérifiée en calculant les cœfficients de corrélation entre chacune de ses parties et son score global. Les cœfficients « Pearson » observés entre les six variables dépendantes considérées et la totalité de ces variables fluctuent entre 0,6288 et 0,8492 . Il est donc permis de conclure à la validité de l'outil, prenant en outre en considération que les règles d'usage en ce qui a trait aux prétests ont été suivies.

Les données furent comparées à l'aide du test « $t$ » de Student, puis analysées successivement en utilisant une analyse discriminante et une régression 
multiple. L'effet des variables intermédiaires, comme le revenu familial annuel ou la scolarité, fut évalué en contrôlant leur impact sur d'autres variables, en particulier la variable indépendante de la taille.

\section{Hypothèse}

L'hypothèse principale de cette recherche est la suivante : les cadres des organisations de petites ou moyennes dimensions (de 1 à 500 employés) démontrent un niveau de satisfaction distinct de celui de leurs collègues évoluant dans des entreprises de grande taille (501 employés et plus). Plus spécifiquement, les premiers (œuvrant en contexte de PME) par rapport aux seconds seront plus satisfaits :

- de la vision qui leur est proposée à l'égard de leur milieu de travail,

- de l'éthique administrative véhiculée,

- du perfectionnement mis à leur disposition,

- de leurs choix éventuels de carrière,

- du système de support en vigueur,

- des conditions dans lesquelles ils fournissent leur prestation de travail.

\section{Cadre de référence}

Les variables où des écarts furent observés concernent six champs distincts et sont définies ci-après.

1. La vision réfère à une manière de voir, de concevoir ou de comprendre l'organisation, à une image atteignable du futur. Elle s'applique soit à l'organisation dans laquelle se situe le cadre, soit au réseau " social » auquel appartient l'établissement pour lequel le cadre travaille. En outre, elle implique deux niveaux d'autorité : d'abord la vision des dirigeants et subséquemment l'adhésion de leurs subalternes à cette vision.

2. L'éthique administrative se traduit surtout par un ensemble de règles de conduite. Elle concerne trois éléments : la perception par les cadres que les décisions prises à leur endroit sont moralement justifiées ; l'idée qu'il y ait peu de favoritisme dans le milieu ; la confidentialité démontrée lorsqu'il s'agit de gérer les dossiers de la clientèle. 
3. Le perfectionnement est lié à l'intérêt démontré pour l'amélioration des habiletés et des attitudes du personnel en vue d'assurer l'avenir de l'organisation. Il a été évalué à l'aide de deux composantes : l'accès au perfectionnement de courte durée et le développement des connaissances par le travail.

4. Le support reçu à l'interne réfère d'abord à l'entraide existant entre collègues de travail et, par la suite, aux marques de considération du supérieur hiérarchique à l'égard de la contribution d'un individu dans le site de travail.

5. Un seul descripteur porte sur le cheminement professionnel futur, soit la possibilité d'exercer éventuellement des choix de carrière.

6. Finalement, les conditions dans lesquelles le cadre fournit sa prestation de travail concerne l'horaire de l'emploi ou les exigences formelles du milieu à l'égard de modes d'organisation du travail.

Il n'y a pas de consensus sur la définition d'une organisation publique de taille réduite (PME). Compte tenu de la nature du secteur précité et des études préalablement considérées, la démarcation fut établie à 500 employés. Une telle approche dichotomisée permet de constater que l'effet de la taille concerne six variables fondamentales de la satisfaction au travail. Un dirigeant de PME par rapport à son collègue de la grande entreprise parvient mieux à inspirer ses subalternes (vision), à faire accepter ses décisions (éthique), à faire en sorte que ses ressources humaines relèvent les défis de leur fonction (perfectionnement) dans un climat de partage et d'appui (support interne). Les cadres des organisations de petite ou moyenne dimension croient davantage que ceux des grands établissements que leur avenir professionnel est potentiellement prometteur (carrière) et que la réalisation de leur prestation d'emploi est stimulante quoiqu'exigeante (conditions de travail). Il ressort que quatre variables intermédiaires affectent toutefois les effets de la taille soient deux au plan personnel (scolarité et revenu familial) et deux au niveau organisationnel (le nombre de griefs et le directorat).

La figure 1 (page suivante) présente l'agencement des variables où des écarts intergroupes sont observés.

\section{Les résultats}

Des différences significatives sont notées au niveau de dix-huit descripteurs : six représentant des variables sociodémographiques et 12 autres constituant les variables dépendantes. Le tableau 1 (page suivante) présente les différences exprimées entre les deux groupes. 
FIGURE 1

Agencement des variables où des écarts sont observés

\section{VARIABLES INTERMÉDIAIRES}

SOCIODEMO

PERSONNELLES
SOCIODÉMO ORGANISATIONNELLES

\begin{tabular}{|c|c|}
\hline $\begin{array}{l}\text { - Scolarité } \\
\text { - Revenu famillial }\end{array}$ & $\begin{array}{l}\text { - Nombre de griefs } \\
\text { - Directorat }\end{array}$ \\
\hline INDÉPENDANTE & DEPENDANTES \\
\hline $\begin{array}{l}\text { TAILLE } \\
\text { Petite moyenne }=1 \text { a } 500 \\
\text { Grande }=501 \text { et plus }\end{array}$ & $\begin{array}{l}\text { - Vision } \\
\text { - Ethique administrative } \\
\text { - Perfectionnement } \\
\text { - Support interne } \\
\text { - Cheminement de carriere } \\
\text { - Conditions de travail }\end{array}$ \\
\hline
\end{tabular}

TABLEAU 1

Écarts observés entre les cadres des organisations de petite ou moyenne taille (PME) et ceux de grande taille (GT)

PME : de 1 à 500 employés

GT : 501 employés et plus

$$
\mathrm{N}=691
$$

\begin{tabular}{|c|c|c|c|c|}
\hline & $\begin{array}{l}\text { Moyenne } \\
\text { PME/GT }\end{array}$ & $\begin{array}{l}\text { Ecart type } \\
\text { PME/GT }\end{array}$ & $\begin{array}{l}\text { Test } \\
\ll t »\end{array}$ & $\begin{array}{l}\text { Nombre } \\
\text { PME/GI }\end{array}$ \\
\hline $\begin{array}{l}\text { VARIABLES } \\
\text { INTERMÉDIAIRES } \\
\text { 1. Scolarité (années) } \\
\text { 2. Groupe d'âge } \\
3=36-45 \text { ans } \\
4=46-55 \text { ans } \\
\text { 3. Revenu familial annuel } \\
\text { (en milliers) } \\
\text { 4. Nombre de syndicats } \\
\text { dans le service (de 1à 6) } \\
\text { 5. Ancienneté réseau } \\
3=11-15 \text { ans } \\
4=16-20 \text { ans } \\
\text { 6. Statut d'emploi } \\
1=\text { T.C. } \\
2=\text { T.P. }\end{array}$ & $\begin{array}{l}16,50 / 15,80 \\
3,00 / 3,30 \\
0,43 / 0,46 \\
2,50 / 2,90 \\
3,74 / 4,18 \\
1,50 / 1,60\end{array}$ & $\begin{array}{l}2,50 / 2,50 \\
0,76 / 0,85 \\
1,00 / 1,00 \\
1,03 / 1,13 \\
1,39 / 1,47 \\
0,49 / 0,48\end{array}$ & $\begin{array}{l}3,18 * * \\
-3,58 * * * \\
-1,84 * \\
-4,15 * * * \\
-3,99 * * *\end{array}$ & $\begin{array}{l}277 / 405 \\
281 / 410 \\
203 / 273 \\
278 / 395 \\
280 / 407 \\
277 / 396\end{array}$ \\
\hline
\end{tabular}

Revue internationale P.M.E., vol. 5, $\mathrm{n}^{\circ} 2,1992$ 


\begin{tabular}{|c|c|c|c|c|}
\hline & $\begin{array}{l}\text { Moyenne } \\
\text { PME/GT }\end{array}$ & $\begin{array}{l}\text { Ecant type } \\
\text { PME/GT }\end{array}$ & $\begin{array}{l}\text { Test } \\
\alpha t »\end{array}$ & $\begin{array}{l}\text { Nombre } \\
\text { PME/GT }\end{array}$ \\
\hline $\begin{array}{l}\text { VARIABLES DEPENDANTES } \\
\quad 5=\text { satisfait, } \\
1=\text { insatisfait } \\
\text { 1. VISION }\end{array}$ & & & & \\
\hline $\begin{array}{l}1.1 \text { Vision de l'employeur } \\
\text { à l'égard du réseau }\end{array}$ & $3,57 / 3,35$ & $1,07 / 1,04$ & $2,68 * *$ & $277 / 409$ \\
\hline $\begin{array}{l}1.2 \text { Vision du supérieur } \\
\text { immédiat à l'égard } \\
\text { de l'organisation }\end{array}$ & $3,31 / 3,01$ & $0,99 / 0,96$ & $3,87 * * *$ & $274 / 403$ \\
\hline $\begin{array}{l}1.3 \text { Adhésion du cadre } \\
\text { à la vision proposée } \\
\text { 2. ÉTHIQUE ADMINISTRATIVE }\end{array}$ & $3,09 / 2,92$ & $1,06 / 1,09$ & $1,98 * *$ & $281 / 409$ \\
\hline $\begin{array}{l}2.1 \text { Perception que } \\
\text { les décisions sont } \\
\text { moralement justifiées }\end{array}$ & $3,41 / 3,16$ & $1,03 / 1,09$ & $2,92 * *$ & $279 / 408$ \\
\hline 2.2 Absence de favoritisme & $2,95 / 2,76$ & $1,17 / 1,14$ & $2,15^{*}$ & $280 / 409$ \\
\hline $\begin{array}{l}\text { 2.3 Confidentialité des } \\
\text { dossiers la clientèle }\end{array}$ & $3,24 / 3,09$ & $1,02 / 1,10$ & $1,91^{*}$ & $\begin{array}{c}281 / 410 \\
.\end{array}$ \\
\hline $\begin{array}{l}3.1 \text { Accès au perfection- } \\
\text { nement de courte durée }\end{array}$ & $3,27 / 3,04$ & $1,11 / 1,09$ & $2,61^{*}$ & $279 / 410$ \\
\hline $\begin{array}{l}\text { 3.2 Développement } \\
\text { des connaissances } \\
\text { par le travail }\end{array}$ & $3,03 / 2,78$ & $1,27 / 1,14$ & $2,69 * *$ & $280 / 407$ \\
\hline $\begin{array}{l}\text { 4. SUPPORT } \\
\text { ORGANISATIONNEL }\end{array}$ & $2,54 / 2,36$ & $1,13 / 1,10$ & $2,11^{*}$ & $280 / 408$ \\
\hline $\begin{array}{l}\text { 5. CHOIX EVENTUEL } \\
\text { DE CARRIERE } \\
\text { 6. CONDITIONS DE TRAVAII }\end{array}$ & $2,88 / 2,72$ & $1,09 / 1,06$ & $1,91^{*}$ & $271 / 395$ \\
\hline 6.1 Horaire de travail & $4,05 / 4,20$ & $1,00 / 0,91$ & $-1,94^{*}$ & $281 / 410$ \\
\hline $\begin{array}{l}6.2 \text { Exigences formelles } \\
\text { du milieu }\end{array}$ & $3,40 / 3,22$ & $0,85 / 0,96$ & $2,57 * *$ & $279 / 408$ \\
\hline
\end{tabular}

$*=\mathrm{P}<.05, \quad * *=\mathrm{P}<.01, \quad * * *=\mathrm{P}<.001$

Au plan sociopersonnel, les cadres des PME publiques du secteur sous étude sont, par rapport à ceux des grandes entreprises, plus scolarisés, plus jeunes et déclarent un revenu familial annuel moins élevé. Ils possèdent moins d'ancienneté dans leur réseau d'appartenance et travaillent davantage à plein temps. Cet écart est principalement dû au fait que les centres hospitaliers comptent des coordonnatrices de nuit à temps partiel. Finalement, ils gèrent moins en contexte de «pluralisation » des unités syndicales. En effet, dans la 
grande organisation, il est observé qu'un nombre plus élevé d'associations représentatives sont présentes dans le site de travail.

Une quantité appréciable de nouvelles organisations publiques ont été créées au Québec au cours de la dernière décennie, spécialement les Centres locaux de santé communautaire (CLSC). Le type de main-d'œuvre requis explique l'essentiel des différences sociodémographiques observées. En effet, les cadres des grandes entreprises œuvrent principalement dans les hôpitaux construits très antérieurement aux CLSC.

Un premier regard sur les résultats confirme l'hypothèse générale voulant que la satisfaction au travail des cadres des PME publiques soit plus élevée que celle de leurs collègues des grands établissements. Globalement, les cadres des PME évaluent que leur employeur leur offre une vision plus stimulante de la raison d'être de leur organisation de telle sorte qu'il leur est plus facile d'y adhérer. Dans la même foulée, la direction est perçue comme étant davantage éthique dans ses actions.

Le perfectionnement des ressources humaines est mieux apprécié en contexte de PME. La relation entre l'exécution du travail et le développement des connaissances apparaît plus tangible chez les cadres des petites ou moyennes organisations. L'impression d'exercer un meilleur contrôle sur le développement futur de sa carrière est ressentie davantage dans les milieux à taille restreinte où le gestionnaire se sent d'ailleurs mieux soutenu à l'interne dans l'exercice de son emploi, soit par son supérieur, soit par ses collègues.

Le tableau 2 indique le classement des variables selon la valeur de la corrélation canonique trouvée à la suite d'une analyse discriminante. Chaque dimension sera analysée en regard des résultats du tableau 1 sur les différences se rapportant à la taille.

TABLEAU 2

Classement des variables dépendantes à la suite d'une analyse discriminante (Cœfficient de corrélation canonique)

VARIABLES

$\begin{array}{ll}\text { 1. Vision } & 0,87 \\ \text { 2. Éthique administrative } & 0,75 \\ \text { 3. Perfectionnement } & 0,65 \\ \text { 4. Support interne } & 0,55 \\ \text { 5. Choix éventuels de carrière } & 0,50 \\ \text { 6. Conditions de travail } & 0,12\end{array}$


Le premier facteur, soit la vision, différencie grandement les deux groupes. Cela est particulièrement évident pour la vision du supérieur immédiat à l'égard de l'avenir de l'organisation (test $t=3,87^{* * *}$ ). La même observation s'applique à la vision de l'employeur à l'égard du réseau où évolue l'organisation (test $t=2,68^{* *}$ ). Le cadre de la PME publique vit une situation de partage d'informations et d'activités qui suscite son adhésion aux deux niveaux de vision précités (test $t=1,98^{* *}$ ).

Le second facteur concerne l'éthique administrative. Dans les établissements de petite et moyenne dimension, les décisions semblent mieux justifiées (test $t=2,92^{* *}$ ) et prises dans un climat qui apparaît moins teinté de favoritisme (test $\mathrm{t}=2,15^{*}$ ). Il y a aussi lieu de préciser que la PME publique dégage un meilleur respect de la confidentialité dans la gestion des dossiers de la clientèle (test $\mathrm{t}=1,91^{*}$ ), ceux-ci ayant à franchir peu d'étapes et, par conséquent, étant en fin de compte traités par moins d'individus.

Le troisième facteur réfère au perfectionnement. D'abord, les cadres des PME accordent un rôle accru à leur travail comme moyen pratique de développement des connaissances (test $\mathrm{t}=2,69^{* *}$ ) et les activités de développement dans lesquelles ils s'inscrivent sont davantage de courte durée (test $t=2,61^{*}$ ). En fait, toute absence d'un cadre dans une PME crée généralement une pression accrue sur l'équipe qui invite la personne en congé à reprendre ses activités régulières de travail le plus tôt possible.

La quatrième variable est le support interne. Dans cette dynamique de gestion intégrée propre aux entreprises de taille réduite, chacun doit obligatoirement compter sur l'autre. Il est donc dans l'ordre des choses que les cadres des PME se sentent mieux appuyés par leurs collègues ou leurs patrons et estiment recevoir davantage d'aide dans l'exécution régulière de leur fonction (test $\mathrm{t}=2,11^{*}$ ).

Un seul descripteur concernait le développement de carrière. En fait, les cadres des PME estiment qu'ils pourront réaliser de meilleurs choix au cours de leur carrière (test $t=1,91^{*}$ ). Ils évoluent sur des fonctions moins rémunérées et sont davantage scolarisés que leurs collègues des grands établissements. Leur potentiel d'acquisition de nouvelles fonctions est donc en principe plus élevé.

La dernière variable porte sur les conditions dans lesquelles un cadre effectue sa prestation de travail. À cet égard, l'hypothèse subsidiaire posée n'est que partiellement confirmée. Comparé à la situation dans les organisations de grande taille, le travail en établissement de dimension réduite exige que moins d'individus prennent charge des exigences des horaires sur différents quarts. Comme les possibilités de délégation à des paliers subalternes sont plus faibles, la pression ressentie par le cadre est donc plus forte. Ainsi, le cadre de la PME publique est moins satisfait de son horaire de travail (test $t=-1,94^{*}$ ), quoique 
dans l'ensemble, ce dernier perçoit plus favorablement les exigences formelles de son milieu sur les façons d'exécuter sa prestation d'emploi (test $t=2,57^{* *}$ ). En clair, les exigences de la clientèle ne lui laissent guère le choix quant à une présence intense et souvent « entrecoupée » dans l'organisation. Toutefois, il a l'impression d'exercer une meilleure influence sur les résultats de son travail.

L'effet des variables intermédiaires fut contrôlé à l'aide d'une régression multiple avec la méthode « Stepwise ». Plusieurs variables intermédiaires furent testées, spécialement les catégories d'établissements, ainsi que les répartitions des répondants selon l'âge, le sexe, le statut d'emploi ou la taille du service. Les cœfficients « Beta » indiquent que quatre variables affectent la relation exprimée par les hypothèses. Ces variables sont la scolarité, le revenu familial annuel, le directorat et le nombre de griefs reçus par le cadre dans l'exercice de ses responsabilités.

L'influence des facteurs sociodémographiques est réelle, quoique peu intense. Ils affectent le support interne, les choix de carrière et les conditions de travail. Aucun effet n'est observé sur la vision, l'éthique administrative et le perfectionnement.

Le tableau 3 présente l'effet des variables sociodémographiques sur les variables dépendantes.

TABLEAU 3

Relations entre les variables intermédiaires et les variables dépendantes

\begin{tabular}{|l|c|c|c|}
\hline & $\begin{array}{c}\text { Support } \\
\text { interne }\end{array}$ & $\begin{array}{c}\text { Choix } \\
\text { de carrière }\end{array}$ & $\begin{array}{c}\text { Conditions } \\
\text { de travail }\end{array}$ \\
\hline - Scolarité & $.11^{*}$ & - & - \\
- Revenu familial annuel & - & $.08^{*}$ & - \\
- Directorat (hiérarchique vs conseil) & - & - & $.13^{*}$ \\
- Nombre de griefs (depuis 2 ans) & - & - & $.10^{*}$ \\
\hline
\end{tabular}

$*=\mathrm{P}<.05$

Le support ou l'appui psychologique reçu par le cadre à l'interne dans l'exécution de son travail est influencé par la scolarité. Spécialement dans la moyenne entreprise ( 250 - 500 employés), là où il y a souvent plus d'un niveau d'autorité, les cadres détenant un emploi plus élevé dans la hiérarchie sont plus scolarisés. Ils estiment recevoir davantage d'appui que ceux évoluant immédiatement au-dessus des salariés. Il faut préciser que ces mêmes cadres reçoivent plus fréquemment un boni annuel au rendement que leurs collègues évoluant au niveau équivalent à celui de contremaître. 
Les choix de carrière fluctuent en fonction du revenu familial. L'intention de mobilité organisationnelle future diminue avec l'accroissement du revenu annuel des deux conjoints. Généralement, le besoin d'un emploi plus rémunérateur diminue si le revenu personnel s'intègre à un revenu familial élevé. En outre, la décision d'une personne d'accepter une autre fonction impliquant un déménagement est habituellement prise en tenant compte de la carrière du conjoint. Les femmes expriment des attentes moins vives que les hommes à l'égard de leur promotion future dans leur réseau professionnel d'appartenance. Par rapport aux hommes, elles estiment leurs possibilités d'avancement intraorganisationnel comme étant relativement présentes et accessibles. Une promotion à l'intérieur de l'entreprise a l'avantage d'éviter un déménagement ou une relocalisation des membres de la famille. Il faut préciser qu'il s'agit du réseau de la santé et que la ligne de promotion spécialement visible est celle des directions de soins infirmiers occupés majoritairement par des femmes professionnelles.

La perception des conditions de travail varie selon le directorat et le nombre de griefs reçus. Les cadres des services cliniques qui dirigent les employés évaluent qu'ils doivent se soumettre à des exigences plus intenses que leurs collègues qui en supervisent peu ou pas, tels les cadres conseils. Par conséquent, les griefs qu'ils doivent régler sont évidemment plus fréquents. Les deux descripteurs précités augmentent la contrainte imposée par les activités quotidiennes de travail sur les cadres hiérarchiques.

\section{Conclusion}

Les organisations publiques québécoises possèdent leur propre spécificité dimensionnelle, bien qu'un régime de normes sophistiquées leur soit imposé sous forme de lois, de politiques, de règlements ou de procédures diverses. Dans ce contexte, chaque catégorie d'établissements élabore son propre régime de relations de travail. De plus, chacun détient en quelque sorte son propre schéma comportemento-perceptuel. Ainsi, le niveau de satisfaction au travail est plus élevé dans les organisations à taille réduite malgré un horaire de travail plus contraignant.

Il s'avère que, dans le secteur public, les organisations de petite ou moyenne taille présentent des différences par rapport aux organisations de grande taille au plan de la satisfaction au travail. Toutefois, ces différences se limitent surtout à des variables sociodémographiques (âge-ancienneté) et aux relations avec le supérieur. Certes, des écarts existent pour d'autres composantes de la satisfaction au travail, mais ils sont plutôt minimes. 
La spécificité de la PME publique semble réelle malgré les conditions étatiques d'emploi. Pour la présente étude, nous avons constaté une partie de cette spécificité au plan de la satisfaction des cadres à l'égard d'un ensemble de facteurs liés au travail.

Dans le secteur public québécois, une quantité appréciable de réalités observables chez les organisations de petites et moyennes dimensions le sont également au niveau des entreprises de grande taille. Cela est probablement aussi le cas dans une moindre mesure, dans le secteur privé de l'économie. Ainsi, dans le milieu enquêté, aucun écart intergroupes significatif de satisfaction n'est apparu pour d'autres éléments tels que les conditions physiques de travail, la sécurité d'emploi, les congés ou les avantages sociaux.

Nonobstant un cadre juridique national, les organisations publiques québécoises possèdent chacune leur conseil d'administration respectif avec ses pouvoirs formels. Il y aurait lieu de poursuivre la réflexion sur l'effet de la taille dans ce secteur au niveau de composantes additionnelles et complémentaires comme les pratiques de relations industrielles, les stratégies de management ou la qualité des services à la clientèle. Il y a là un domaine d'investigation susceptible de contribuer à l'avancement de la science administrative.

\section{Bibliographie}

ARTHUR, D. (1987), Managing Human Ressources in Small and Mid-Sized Companies,New York, Amacon Press, 277 p.

BaUmback, C.M. et K. LaWYeR (1979), « How to Organize and Operate a Small Business », Englewood Cliffs, Prentice-Hall Inc., 577 p.

BENoIT-RousseAu, M.D. (1990), « La gestion des ressources humaines dans les petites et moyennes entreprises au Québec », Revue Internationale de P.M.E., vol. 3, $\mathrm{n}^{\circ} 1$, p. $40-55$.

BLUMENTHAL, R.A. (1985), « Management training in the small business setting 》, dans $30^{\circ}$ Conférence mondiale du conseil international de la petite entreprise, Montréal, juin, p. 77-90.

BusKIRK, R.H. et P.J. VAughN (1976), Managing New Enterprises, New York, West Publishing Co., $483 \mathrm{p}$.

Cohn, T. et R.A. Lindberg (1984), Practical Personnel Policies for Small Business, New York, Van Nostrand Reinhold Pub., 216 p.

FILION, L.J. (1990), «L'intrapreneur : un visionnant », Revue Internationale de gestion des petites et moyennes organisations, vol. $5, \mathrm{n}^{\circ} 1, \mathrm{p} .22-32$. 
FILION, L.J. (1991), «Vision and relations : élements for an entrepreneurial metamodel », International Small Business Journal, vol. 9, n² 2, p. 26-40.

HaYLeS, W.D. et R.T. HubBaRd (1983), Small Business Management, New York, Delmar Publishers Inc., 408 p.

HAWBRICK, D.C. et L.M. CROZIER (1984), « Strumblers and stars in the management of rapid growth », Journal of Business Venturing, vol. 1, $\mathrm{n}^{\circ} 1$, p. 31-45.

JoYCE, P., A. Woods, T. MCNulTY et P. CoRRIGAN (1990), « Barriers to change in small business », International Small Business Journal, vol. 8, n 4, p. 49-58.

KET DE VRIES, M.F.R. (1989), « L'envie, grande oubliée des facteurs de motivation en gestion », Gestion, vol. 14, n², mai, p. 10-19.

LAROUCHE, V. et F. DELORME (1975), «Inventaire de satisfaction au travail : validation », Relations Industrielles, vol. 27, $\mathrm{n}^{\circ} 3, \mathrm{p} .343-374$.

LELOUARN, J.Y. (1982), « Les professeurs d'université et leur travail », Relations Industrielles, vol. $37, \mathrm{n}^{\circ} 2,1982$, p. 385-402.

LOCKE, E.A. (1976), « The nature and causes of job satisfaction », dans M.D. Dunnette, Handbook of Industrial and Organizational Psychology, Chicago, Rand McNally.

MAhe DE Boislandelle, H. (1988), Gestion des ressources humaines dans les PME, Paris, Collection Technique de gestion, Économica, 322 p.

McFarlane, W.N. (1977), Principles of Small Business Management, New York, McGraw-Hill Inc., 534 p.

Novick, M.R. (1967), « Coefficient Alpha and the reliability of composite measurements », Psychometrika, vol. 32, p. 1-13.

RAM, M. (1991), « The dynamics of workplace relations in small firms », International Small Business Journal, vol. 10, $\mathrm{n}^{\circ} 1, \mathrm{p}$. 44-53.

RINLER, G.W. et N.J. HUMPHREYS (1980), Small Business : Developping the Winning Mangement Team, New York, Amacon Press, 180 p.

RoXe, L.A. (1979), Personnel Mangement for the Smaller Company, New York, Amacon Press, $246 \mathrm{p}$.

SIVETAR, M. (1985), « SMR forum : entrepreneurs, chaos and creativity : can creative people really survive large structure », Sloan Management Review, hiver, p. 55-62.

Thompson, P. (1989), Des compétences pour l'avenir : la petite entreprise et la formation professionnelle au Canada, Toronto, F.C.E.I., 90 p. 